\title{
Early prognosis of low birthweight infants treated for progressive posthaemorrhagic hydrocephalus
}

\author{
R W I COOKE \\ Department of Child Health, Liverpool University
}

SUMMARY Twelve low birthweight preterm infants were treated for progressive posthaemorrhagic hydrocephalus by ventriculoperitoneal diversion of cerebrospinal fluid. At a median postnatal age of 20 months, 6 were moderately or severely retarded. Convulsions in the first week of life were associated with a poorer prognosis and frequent taps to remove cerebrospinal fluid with a better outcome.

Although survival rates of low birthweight infants with severe respiratory problems have improved, intraventricular haemorrhage has become a common complication affecting the quality of survival. An appreciable proportion of survivors develop enlargement of the cerebral ventricles or clinically evident progressive hydrocephalus. ${ }^{1}$ In many ventricular enlargement stops spontaneously or after intermittent drainage of cerebrospinal fluid through a lumbar or ventricular needle tap. ${ }^{2}$ The rest of these infants need surgical placement of ventriculoatrial or ventriculoperitoneal shunts if gross hydrocephaly and eventual death is to be prevented. Over $2 \frac{1}{2}$ years a number of variables related to early prognosis in a group of low birthweight infants treated by ventricular shunt have been examined.

\section{Patients and methods}

Between July 1979 and December 198114 low birthweight infants referred to the regional neonatal intensive care unit at Liverpool Maternity Hospital developed progressive hydrocephalus after intraventricular haemorrhage. During the same period 808 low birthweight infants were admitted to the unit. Of those infants developing intraventricular haemorrhage, 91 died during the neonatal period or before discharge. All but 1 hydrocephalic infant, who developed hydrocephalus at a late stage, were treated at first by intermittent lumbar or ventricular needle drainage of cerebrospinal fluid. Two also received isosorbide. ${ }^{3}$ After discussion between staff and parents, 2 infants were not treated further by insertion of a shunt because of gross neurological deficit. Both died within a few days. The 12 remaining infants form the study group.

Intraventricular haemorrhage was diagnosed and the development of hydrocephalus monitored by echoencephalography via the anterior fontanelle. ${ }^{4}$ The decision on timing of the shunt insertion was made by 1 of 2 paediatric surgeons. All infants had clinically evident hydrocephalus at the time of operation and ultrasound scan appearances were never the sole criterion for operation. No infant was dependent on a ventilator immediately before surgery, although several required short periods of respiratory support afterwards. One infant suffered postoperative cardiorespiratory arrest and recovered only after prolonged resuscitation. This infant was the most severely handicapped at follow up. Other postoperative complications were minor.

The first follow up after discharge from hospital included a full neurological examination, Denver developmental screening test, and Stycar tests of hearing and vision, as appropriate. As disabilities became evident specialist ophthalmic, physiotherapy, and developmental referrals were made. The assessment of outcome used here is a combination of all assessments made of an individual child's progress. The median length of follow up to date is 20.5 months (range 15-42 months). So that the relation of antecedent factors to early outcome of treatment could be examined, the infants were assigned to 1 of 2 groups on the basis of neurological sequelae and developmental delay. The 'better outcome' group (group 1) comprised 6 infants with failures in 1 or no sections of the Denver developmental screening test and with either physical disabilities that did not appear to interfere seriously with normal function or normal findings on neurological examinations. The 'poorer outcome' group (group 2) all had moderate to severe developmental delay in 2 or more sections of the Denver developmental screening test and major 

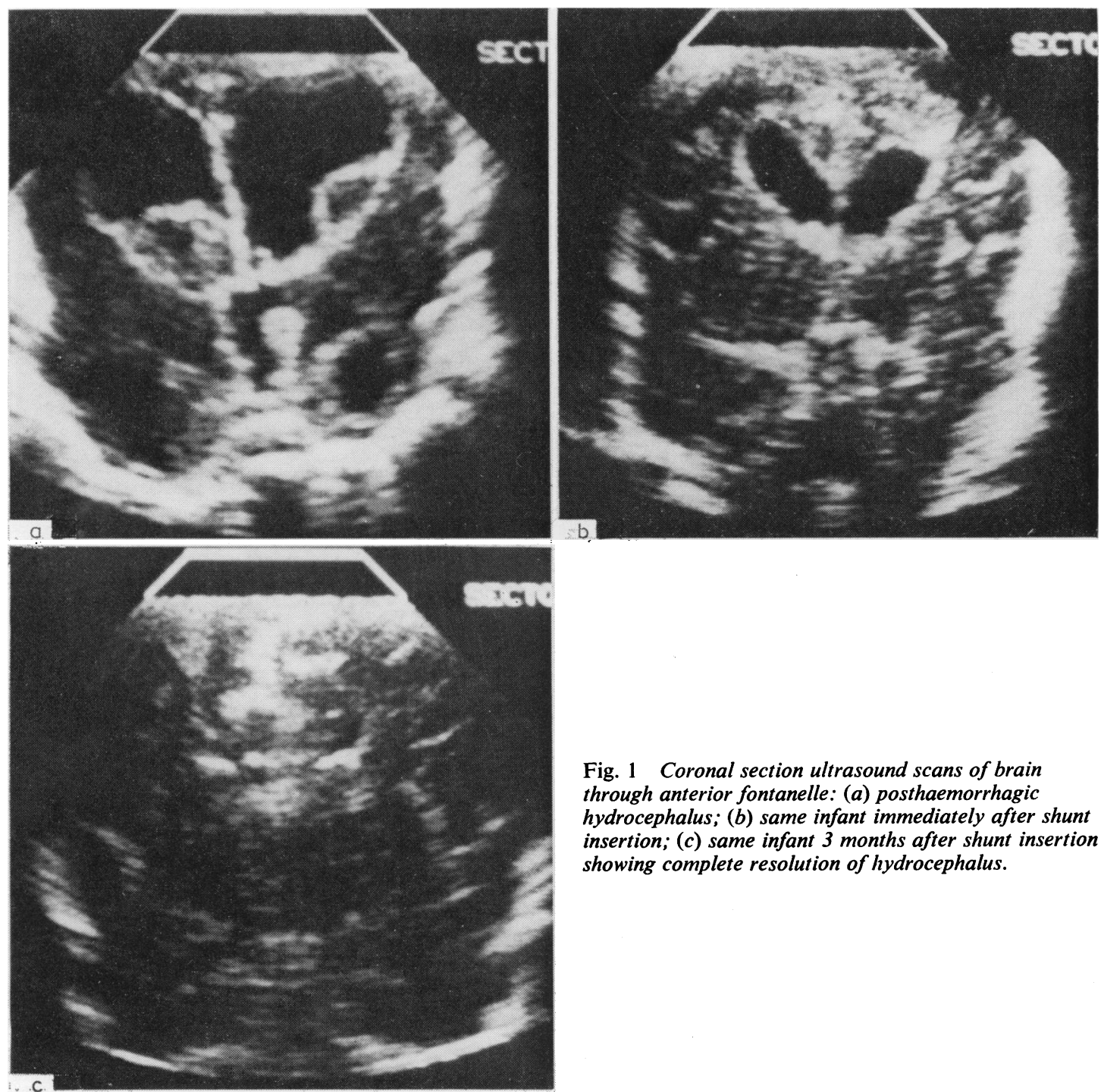

Fig. 1 Coronal section ultrasound scans of brain through anterior fontanelle: (a) posthaemorrhagic hydrocephalus; (b) same infant immediately after shunt insertion; (c) same infant 3 months after shunt insertion showing complete resolution of hydrocephalus.

physical disabilities. The composition of the groups is shown in the Table. Because of the small numbers a further breakdown of outcome was not practicable. Factors possibly related to outcome were analysed by the Fisher exact test or Mann-Whitney U test, as appropriate.

\section{Results}

Ten boys and 2 girls were treated. Both girls were in group 2. The median birthweight was $1295 \mathrm{~g}$ (range 1150-2070 g) and median gestational age was $29 \cdot 5$ weeks (range 27-33 weeks). No infant was small for 

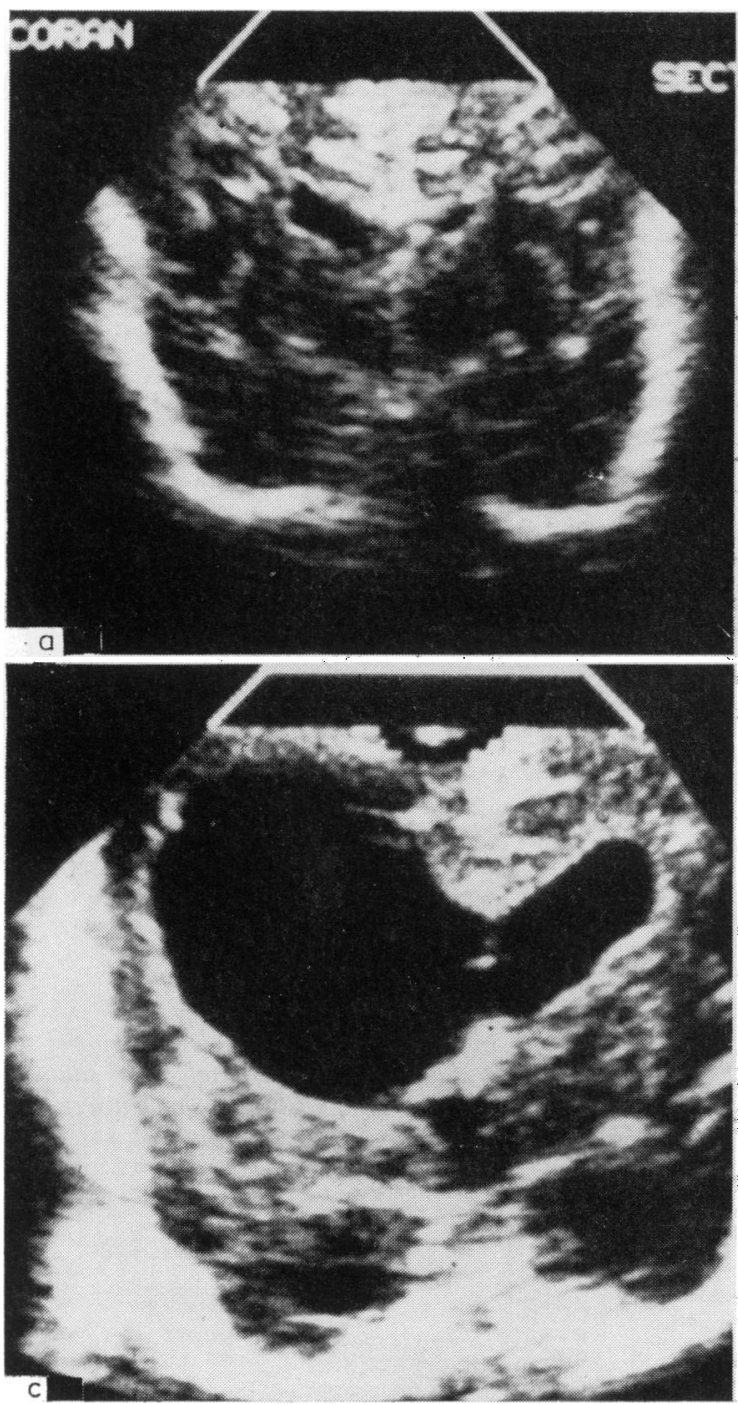

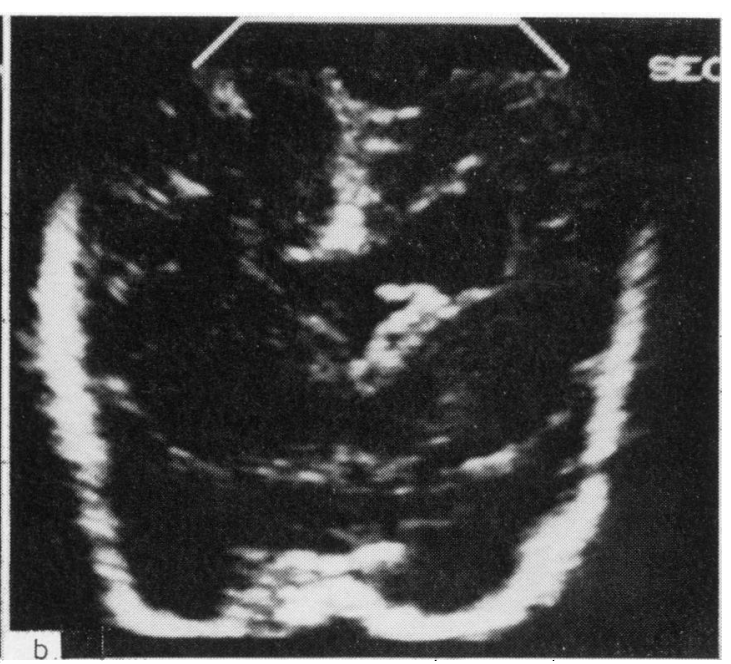

Fig. 2 Post shunt insertion follow up scans: (a) cerebral atrophy in infant with spastic diplegia; (b) porencephalic cyst in normally developing infant; $(c)$ porencephalic cyst in infant with hemiplegia.
Resolution of hydrocephalus, clinically and on ultrasound examination, followed shunt insertion in all cases. In 2 infants the size of the lateral ventricles did not return to normal after shunt insertion and this may have been caused by some periventricular or cerebral atrophy. Four infants required 1 or more replacements of their shunt because of malfunction. In 8 infants porencephalic cysts remained at the site of extraventricular extension of intraventricular haemorrhage.

All infants in group 2 had convulsions in the first week of life. One infant in group 1 had a short fit on the second day. This difference was statistically significant $(P<0 \cdot 025)$. Eleven infants had lumbar or ventricular taps to relieve symptoms of acute hydrocephalus such as recurrent apnoea, vomiting, or fits. The number of taps done was not related to the severity of the condition, as those infants in group 2 had significantly fewer taps (median $=3$, range $1-15 ; P=0.026)$ than those in group 1 (median 14 , range 3-20).

\section{Discussion}

The prognosis of low birthweight infants suffering 


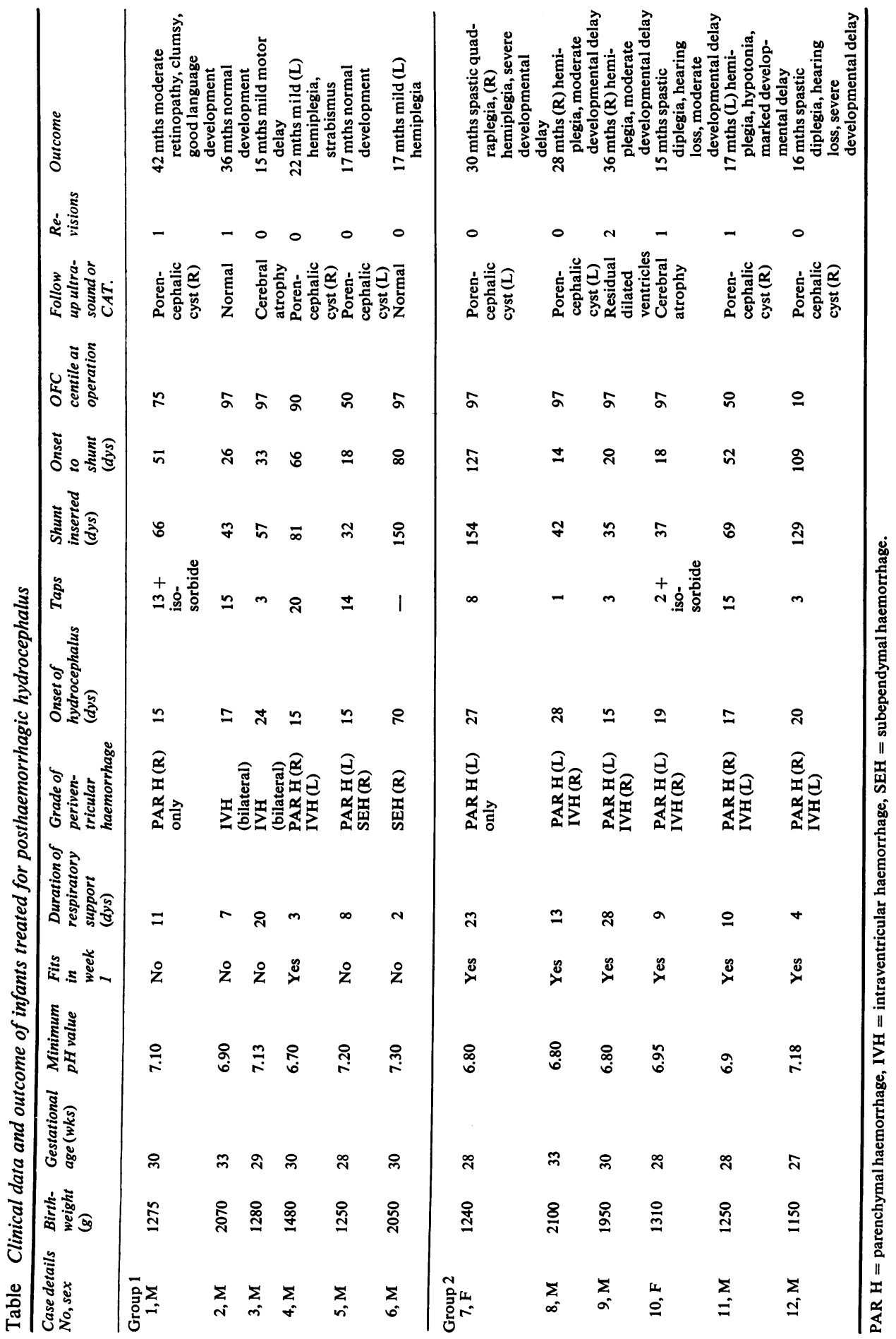


from posthaemorrhagic hydrocephalus is probably related to the extent of the first insult and haemorrhage and the rate and extent of the development of subsequent hydrocephalus. Infants in group 2 were ventilated for longer, suffered more severe acidosis, and more extensive haemorrhage than those in group 1, but these differences were not statistically significant. Early convulsions have been widely recognised as a risk factor for later disability and probably relate to the extent of acute neurological insult in the first few days. ${ }^{5}$ Greater attention to neurological examination at this time could provide more subtle indicators of prognosis. ${ }^{6}$

Although the time of onset of clinical hydrocephalus was fairly uniform, the rate of progression was variable and accounted for the wide differences in the timing of the operation. It has been suggested that hydrocephalus produces further impairment by causing ischaemia of periventricular areas and that lowering intraventricular pressure by intermittent drainage may reduce this. ${ }^{7}$ The 12 infants described here had taps for a variety of reasons, but it is interesting that more frequent taps were associated with a better outcome. Even if ventriculoperitoneal shunts cannot be avoided by repeated tapping, earlier reduction of hydrocephalus warrants further investigation as a means of improving prognosis.
I thank Mr Roger Cudmore and Miss Irene Irving, consultant paediatric surgeons, for advice and the surgical care of these patients.

\section{References}

1 Deonna T, Payot M, Probst A, Prod'hom L-S. Neonatal intracranial hemorrhage in premature infants. Pediatrics 1975; 56: 1056-64.

2 Papile L, Burstein J, Burstein R, Koffler H, Koops BL, Johnson JD. Post Hemorrhagic hydrocephalus in lowbirth weight infants; treatment by serial lumbar punctures. J Pediatr 1980; 97 : 273-7.

3 Lorber J, Bhat US. Post haemorrhagic hydrocephalus: diagnosis, differential diagnosis, treatment, and long term results. Arch Dis Child 1974; 49: 751-62.

4 Cooke RWI. Letter: Ultrasound examination of neonatal heads. Lancet 1979; ii: 38.

5 Keen JH, Lee D. Sequelae of neonatal convulsions: study of 112 infants. Arch Dis Child 1973; 48: 542-6.

6 Dubowitz LMS, Dubowitz V. The neurological assessment of the preterm and full term newborn infant. Clinics in Developmental Medicine No 79, London: Heinemann, 1981.

7 Hill A, Volpe JJ. Decrease in pulsatile flow in the anterior cerebral arteries in infantile hydrocephalus. Pediatrics 1982; 69: 4-7.

Correspondence to Dr R W I Cooke, Department of Child Health, Liverpool Maternity Hospital, Oxford Street, Liverpool L7 7BN.

Received 2 March 1983

\section{Notice to contributors}

Please note that revised guidelines for the submission of manuscripts have been printed inside the front cover of this journal and, in particular, that two copies of papers should be submitted. 